\title{
Association between baseline and changes in high-sensitive C-reactive protein and metabolic syndrome: a nationwide cohort study and meta-analysis
}

Qingping Xue ${ }^{1,2}$, Xue Yang $^{3}$, Yuli Huang ${ }^{4,5}$, Dongshan Zhu ${ }^{6}$, Yi Wang ${ }^{7}$, Ying Wen ${ }^{8}$, Jian Zhao ${ }^{9}$, Yanjun Liu ${ }^{10,11}$, Chun-Xia Yang ${ }^{3}$, Jay Pan ${ }^{2,3}$, Tong Yan ${ }^{11^{*}}$ and Xiong-Fei Pan ${ }^{12^{*}}$ (i)

\begin{abstract}
Background: We aimed to prospectively evaluate the associations between the baseline and changes in high-density C-reactive protein (hs-CRP) and incident metabolic syndrome (MetS) in China and update the evidence based on a meta-analysis of cohort studies in different populations.

Methods: Data from the China Health and Retirement Longitudinal Study among adults aged 45 years or older were analyzed. Participants who were recruited in the study in 2011-2012 without MetS and successfully followed up to 2015-2016 were included in our final analysis. Logistic regressions were applied to examine the prospective associations of baseline and changes in hs-CRP with incident MetS and estimate corresponding odds ratios (ORs) and 95\% confidence intervals $(95 \% \mathrm{Cls}$ ). A meta-analysis was conducted to synthesize effect estimates from our findings and other cohort studies on this topic.
\end{abstract}

Results: Among 4,116 participants, 535 developed MetS after a 4-year follow-up. Compared with the participants with hs-CRP in the lowest quartile, those with hs-CRP in the second, third, and highest quartiles had higher odds of MetS, with multivariable-adjusted ORs $(95 \% \mathrm{Cls})$ of $1.51(1.12,2.06), 1.50(1.11,2.04)$, and $1.83(1.37,2.47)$. For the hsCRP changes, ORs $(95 \%$ Cls) were $3.24(2.51,4.02), 3.34(2.56,4.38)$, and $3.34(2.54,4.40)$ respectively. One unit (log of $1 \mathrm{mg} / \mathrm{L}$ ) increase in hs-CRP was associated with $23 \%$ higher risk of MetS (OR 1.23; 95\% Cl 1.10, 1.38). In a metaanalysis of 6 cohort studies, the pooled relative risk for MetS was $1.63(1.38,1.93)$ for the highest versus lowest level of hs-CRP. In addition, the pooled relative risk for MetS was $1.29(1.05,1.59)$ for each unit increase of hs-CRP after log-transformation.

Conclusions: Both higher baseline hs-CRP and longitudinal hs-CRP increases were associated with higher risks of incident MetS. Individuals with high hs-CRP levels may need to be closely monitored for future risk of MetS.

Keywords: High-density C-reactive protein, Metabolic syndrome, Chinese, Cohort study, Meta-analysis

*Correspondence: ethanmed@yeah.net; pxiongfei@gmail.com

${ }^{11}$ Center for Obesity and Metabolic Health, The Third People's Hospital of Chengdu and The Affiliated Hospital of Southwest Jiaotong University, Chengdu, Sichuan, China

${ }^{12}$ Key Laboratory of Birth Defects and Related Diseases of Women and Children (Sichuan University), Ministry of Education, West China Second University Hospital, Sichuan University, Chengdu, Sichuan, China Full list of author information is available at the end of the article

\section{Background}

Metabolic syndrome (MetS) is characterized by a constellation of interrelated metabolic risk factors, including abdominal obesity, hypertension, high blood glucose levels, and dyslipidemia [1]. It is associated with an approximate twofold increased risk for original author(s) and the source, provide a link to the Creative Commons licence, and indicate if changes were made. The images or other third party material in this article are included in the article's Creative Commons licence, unless indicated otherwise in a credit line to the material. If material is not included in the article's Creative Commons licence and your intended use is not permitted by statutory regulation or exceeds the permitted use, you will need to obtain permission directly from the copyright holder. To view a copy of this licence, visit http://creativecommons.org/licenses/by/4.0/. The Creative Commons Public Domain Dedication waiver (http://creativeco mmons.org/publicdomain/zero/1.0/) applies to the data made available in this article, unless otherwise stated in a credit line to the data. 
cardiovascular disease and a fivefold increased risk for incident type 2 diabetes mellitus [2]. With a rapid increase of MetS worldwide, it is imperative to identify and manage the medical condition in its early stage.

Although the understanding of pathophysiological mechanisms for MetS is evolving, some evidence suggests that inflammation is involved in the development of MetS. High-sensitivity C-reactive protein (hsCRP), known as an acute-phase reactant produced by liver in response to the presence of the pro-inflammation cytokines [3], is positively associated with MetS in several cohort studies in the US [4], European [5] and Asian countries [6,7]. Most extant cohort studies were conducted in non-Chinese populations, and one cohort study among 886 southern Chinese adults with a five-year follow-up found that a high hs-CRP level was related with an increased risk of incident MetS [6]. However, this study was subject to several limitations, such as a small sample size, a narrow sampling frame, and a short follow-up. In addition, most studies on the effects of hs-CRP on cardiometabolic diseases only used a baseline measure of hs-CRP without considering the dynamic changes of hs-CRP. One study from the US showed increased risks of incident diabetes, cardiovascular disease, and mortality in relation to sustained elevated hs-CRP [8], while a separate study from China found that adults with "moderate-increased" hs-CRP (moderate at baseline and increased to high concentration at follow-ups) had a higher risk of incident diabetes, compared with those with "low-stable" hs-CRP (low at baseline and maintained at low concentration at follow-ups) [9]. These findings suggest that longitudinal changes, particularly sustained elevations of hs-CRP may carry extra information for chronic inflammation compared with single hs-CRP measurements. However, no prior studies ever explored the association of longitudinal changes in hsCRP with incident MetS. Given the high prevalence of MetS (24.5\%) in Chinese adults [10], it is necessary to systematically assess the link of hs-CRP to MetS risk using national population-based longitudinal data in China.

Thus, our research aimed to use the data from the China Health and Retirement Longitudinal Study (CHARLS) to prospectively examine the associations of baseline hs-CRP and longitudinal hs-CRP changes with incident MetS among middle-aged and elderly Chinese and assess whether the associations differed in subpopulations. Since prior prospective studies on this topic from different populations contained mixed findings $[11,12]$, we pooled our findings with reports from previous cohort studies to corroborate our work.

\section{Methods}

\section{Study population}

The CHARLS was conducted in a national representative sample of adults aged 45 years or older in China. The study design, sampling procedures and survey methods of the CHARLS were reported in details elsewhere [13]. Briefly, based on a probability-proportional-tosize sampling design, a total of 17,708 participants were recruited from 150 counties of 28 provinces at baseline between June 2011 and March 2012. At enrollment, data were collected by trained staff using standardized questionnaires on demographic and socioeconomic characteristics, health conditions, health-related lifestyle and behaviors, and medical conditions. Physical measurements and blood samples were also obtained at baseline. The participants were followed up until 2015-2016, and similar procedures for data collection were repeated in the 2015-2016 follow-up. Plasma samples were aliquoted and immediately frozen at $-20{ }^{\circ} \mathrm{C}$, and transported to the Chinese Center for Disease Control and Prevention in Beijing within 2 weeks where they were placed in a deep freezer and stored at $-80{ }^{\circ} \mathrm{C}$ until assayed at the Capital Medical University laboratory. The CHARLS was approved by the Biomedical Ethics Review Committee of Peking University (IRB00001052-11,015), and informed consent was obtained from all study participants.

Our analyses utilized prospective data from the CHARLS collected between 2011 and 2016. Among 17,708 participants in the CHARLS, 11,847 completed both questionnaires and laboratory measurements at baseline. In our work, we excluded (1) participants without information for MetS at baseline $(n=2028)$; (2) participants with MetS at baseline $(n=2740)$; (3) participants with hs-CRP $\geq 10 \mathrm{mg} / \mathrm{L}$ at baseline $(\mathrm{n}=276)$ as high levels of hs-CRP could be caused by acute inflammation [14]; (4) participants who were lost to followup ( $\mathrm{n}=2219)$; (5) participants without information of MetS or hs-CRP in 2015-2016 $(\mathrm{n}=87)$; and (6) participants with data missing for age $(n=177)$, residential area $(n=4)$, cigarette smoking $(n=12)$, alcohol drinking $(n=3)$, BMI $(n=33)$, diabetes $(n=60)$, hypertension $(n=19)$, dyslipidemia $(n=53)$, heart diseases $(n=12)$, or stroke $(n=8)$. A total of 4,116 who had no MetS at baseline and were followed up successfully until 2015-2016 were included in final analyses (Additional file 1: Fig. S1).

\section{Definition of MetS}

MetS was diagnosed according to the Chinese guidelines for the management of dyslipidemia in adults [15] as having three or more of the following items: (1) waist circumference $(W C) \geq 90 \mathrm{~cm}$ of men or $\geq 80 \mathrm{~cm}$ of women; (2) fasting blood glucose (FBG) levels $\geq 110 \mathrm{mg} /$ 
$\mathrm{dl}(6.10 \mathrm{mmol} / \mathrm{L})$ or undergoing anti-diabetes treatment; (3) systolic blood pressure (SBP) $\geq 130 \mathrm{~mm} \mathrm{Hg}$ or diastolic blood pressure (DBP) $\geq 85 \mathrm{~mm} \mathrm{Hg}$ or undergoing anti-hypertensive drug treatment; (4) fasting triglycerides (TG) $\geq 150 \mathrm{mg} / \mathrm{dl}(1.7 \mathrm{mmol} / \mathrm{L})$; and (5) fasting highdensity lipoprotein cholesterol levels (HDL-C) $<40 \mathrm{mg} /$ $\mathrm{dl}(1.0 \mathrm{mmol} / \mathrm{L})$. Incident MetS was defined as new-onset MetS during the follow-up in 2015-2016.

\section{Assessment of hs-CRP}

Hs-CRP was measured by the immunoturbidimetric assay at baseline and in the 2015-2016 follow-up, and its analytical range was $0.1-20.0 \mathrm{mg} / \mathrm{L}$ and between-assay coefficient of variation was $<5.7 \%$. The same Roche kits (Roche Diagnostics, Basel, Switzerland) on a Hitachi 7180 chemistry analyzer (Hitachi, Tokyo, Japan) were used for all assays [16].

\section{Measurement of covariates}

Covariates including year of birth, sex, education level (illiterate, primary school, middle school, and high school or above), residence (urban and rural), cigarette smoking (never, former, and current), alcohol drinking (never, former, and current), diabetes (yes and no), hypertension (yes and no), dyslipidemia (yes and no), heart disease (yes and no), and stroke (yes and no) were collected in the baseline survey. BMI was calculated as body weight in kilograms divided by height in meters squared. Prevalent diabetes was determined by fasting glucose measures (FBG $\geq 126 \mathrm{mg} / \mathrm{dl}$ or $\mathrm{HbA} 1 \mathrm{c} \geq 6.5 \%$ ) or self-reported physician diagnosis [17]. Prevalent hypertension was determined by blood pressure $(\mathrm{SBP} \geq 140 \mathrm{mmHg}$ or $\mathrm{DBP} \geq 90 \mathrm{mmHg}$ ) or self-reported physician diagnosis [18]. Prevalent dyslipidemia was defined as total cholesterol/HDL-C $>5$ or self-reported physician diagnosis [19]. Prevalent heart disease and stroke were determined by self-reported physician diagnosis.

\section{Statistical analysis}

Mean (standard deviation, SD) were reported for continuous variables and frequency (percentage, \%) were reported for categorical variables. Analysis of variance (for continuous variables) and Chi-square (for continuous variables) were used to compare basic characteristics in participants across baseline hs-CRP quartiles. Logistic regressions were performed to estimate the odds ratios (ORs) and 95\% confidence intervals (CIs) for the associations between hs-CRP and incident MetS. We adjusted for age (continuous, years), sex, education level, and residence in the first multivariable model, and additionally adjusted for cigarette smoking, alcohol drinking, BMI (continuous, $\mathrm{kg} / \mathrm{m}^{2}$ ), hypertension, dyslipidemia, diabetes, heart disease and stroke in the second multivariable model. In the main analyses, hs-CRP levels were grouped as quartiles and the lowest quartile was set as the reference group. Elevated hs-CRP was defined as a concentration of hs-CRP higher than $3 \mathrm{mg} / \mathrm{L}$ to dichotomize hs-CRP levels [8]. In addition, we used continuous hs-CRP levels with natural log transformation as the independent variable in a separate analysis. Similar analyses were conducted to assess the associations between absolute and percent changes of hs-CRP (group 1 included participants with negative or null changes or percent changes, and group 2-4 were tertiles for the remaining participants) and MetS. The linear trend in the associations of baseline and longitudinal changes in hs-CRP with MetS was assessed by assigning the median hs-CRP or hs-CRP changes of quartiles/groups for logistic regression models. We also assessed the association of combined baseline hs-CRP and absolute hs-CRP changes with incident MetS by segmenting four groups based on whether hs-CRP was higher than $3 \mathrm{mg} / \mathrm{L}$ [8] and the median value of hs-CRP changes: low baseline/low increase (reference group), high baseline/low increase, low baseline/high increase, and high baseline/ high increase.

We conducted two sensitivity analyses to confirm our results. In the first sensitive analysis, we performed multiple imputations for 381 participants with data missing for age, sex, education level, cigarette smoking, residential area, cigarette smoking, alcohol drinking, BMI, diabetes, hypertension, dyslipidemia, heart disease and stroke prior to logistic regressions. Under the assumption of missing at random, we replaced missing values by imputed ones from ten duplicate datasets which were created by imputation simulation to reduce sampling variability. Finally, we combined the effect estimates from the ten imputed datasets to obtain the pooled effect estimates and their 95\% CIs for the associations of both baseline hs-CRP and hs-CRP changes with incident MetS. In the second sensitive analysis, we conducted logistic regressions by excluding participants with diabetes, heart disease and stroke.

We stratified participants by age $(\leq 60$ years and $>60$ years), sex (male and female) and BMI $(<24$ and $\geq 24 \mathrm{~kg} / \mathrm{m}^{2}$ based on the Chinese criteria for overweight and obesity [20]) and added a product term of the stratifying variables and baseline hs-CRP or hsCRP changes to the final model to examine potential effect modifications (interactions) using a likelihood ratio test. 


\section{Meta-analysis}

We conducted a meta-analysis to synthesize our findings with those from previous cohort studies that dealt with this topic. We carried out an electronic search in PubMed and Embase for cohort studies up to May 13, 2020, using a search strategy that combined $\mathrm{MeSH}$ terms and keywords for "metabolic syndrome", "CRP" and "C-reactive protein" (Additional file 1: Table S1). Studies were included for analyses if they met these criteria: 1) they were prospective cohort studies from general population or from patients without MetS at baseline; 2) adjusted relative risks (RRs), ORs, or hazard ratios with $95 \%$ CIs were reported for the prospective association between hs-CRP and incident MetS; and 3 ) the reported language was English. If the same topic was examined in multiple articles that were derived from the same cohort, only the latest published article was included for meta-analysis. The title, abstract, and full text of all identified studies were screened by two investigators (XY and QX) independently (Additional file 1: Fig. S2). Any discrepancies were resolved through discussions to reach a consensus. The NewcastleOttawa quality assessment scale [21] was used to assess the quality of included studies, which is primarily based on the selection of the study groups, the comparability of the groups, and the ascertainment of either the exposure or outcome of interest.

For data synthesis, basic characteristics and fully adjusted effect estimates were extracted from each study. Statistical heterogeneity was indicated by $P<0.01$ in the $\mathrm{Q}$ test. Random-effects (if heterogeneity existed) or fixed-effects (if no heterogeneity existed) meta-analysis was conducted to pool RRs for the highest hs-CRP level category compared with the lowest hs-CRP level category or hs-CRP with natural log-transformation. For studies which reported separate results for men and women, we applied random-effect meta-analysis to combine the effect estimates to obtain total effect prior to final meta-analysis. In our study, we converted the ORs into RRs using the formula $(R R=O R /$ $([1-\mathrm{pRef}]+[\mathrm{pRef} \times \mathrm{OR}])$, where $\mathrm{pRef}$ is the prevalence of the outcome in the reference group [22]. Hazard ratios were regarded as approximate RRs for the meta-analysis. Sensitivity analyses were conducted to explore the influence of individual studies on the summary RRs. We used the funnel plot and Egger's test to assess the publication bias. All statistical analyses were conducted in $\mathrm{R}$ (version 3.6.1) and $P$-value $<0.05$ was considered to indicate statistical significance.

\section{Results}

Baseline characteristics

Of 4,116 participants without MetS at baseline, 53.13\% were male and the mean age was 58.58 (SD, 9.03) years. The median (interquartile range) of hs-CRP was $0.81 \mathrm{mg} /$ dl $(0.48,1.63)$ in our study, $0.76 \mathrm{mg} / \mathrm{dl}(0.45,1.50)$ in females and $0.89 \mathrm{mg} / \mathrm{dl}(0.51,1.78)$ in males. Compared with the lowest quartile of hs-CRP, those in the highest quartile of hs-CRP were more likely to be female, older, urban residents, smokers, and have higher $\mathrm{BMI}, \mathrm{WC}$, FBG, diastolic BP, and TG but lower HDL-C $(P \leq 0.02$ for all; Table 1). In addition, they were more likely to have prevalent heart disease, hypertension, and dyslipidemia $(P \leq 0.04$ for all). There were no major differences in most baseline characteristics between the included 4,116 participants and 2,687 with data missing (Additional file 1: Table S2).

\section{Association of baseline hs-CRP and hs-CRP changes with incident MetS}

During a four-year follow-up, 535 out of 4,116 (13.00\%) participants developed incident MetS. Compared with participants in the lowest quartile of hs-CRP, participants with hs-CRP level in the second, third and fourth quartiles were $1.51(1.12,2.06), 1.50(1.11,2.04)$ and 1.83 (1.37, 2.47; Table 2) times as likely to have MetS after adjustment for potential confounders. There was a linear trend across the four quartiles of hs-CRP ( $P$ for trend $<0.001$; Table 2). Using hs-CRP as a continuous variable with natural log transformation also showed a positive association between hs-CRP levels and incident MetS (multivariable-adjusted OR, 1.23; 95\% CI, 1.10, 1.38; Table 2). Participants with elevated hs-CRP (3 mg/L or higher) showed a $50 \%(1.50 ; 1.14,1.94$; Table 2$)$ higher odds of having MetS compared with those with lower hsCRP after adjustment for sociodemographic factors, but this association became non-significant after additional adjustment for lifestyle and behavior factors and other related diseases. There were no heterogeneities across age, sex and BMI subgroups ( $P$ for interaction $\geq 0.41$; Table 3). In addition, multiple imputations for missing data did not show appreciable changes in effect estimates across four quartiles (Additional file 1: Table S3). Furthermore, the effect estimates across four quartiles remained similar after excluding participants with diabetes, heart disease and stroke (Additional file 1: Table S4).

Compared with participants in the lowest group of absolute hs-CRP changes, those with absolute hs-CRP changes in the second, third and fourth groups were 3.24 
Table 1 Baseline characteristics of study participants in the CHARLS $(N=4116)$

\begin{tabular}{|c|c|c|c|c|c|c|}
\hline \multirow[t]{2}{*}{ Characteristics } & \multirow{2}{*}{$\begin{array}{l}\text { Total } \\
\text { (\%) }\end{array}$} & \multicolumn{4}{|c|}{ Quartiles of hs-CRP (mg/L) } & \multirow[t]{2}{*}{$P$ values } \\
\hline & & $\begin{array}{l}\text { Quartile } 1 \\
(<0.48)\end{array}$ & $\begin{array}{l}\text { Quartile } 2 \\
(\geq 0.48 \text { to }<0.81)\end{array}$ & $\begin{array}{l}\text { Quartile } 3 \\
(\geq 0.81 \text { to }<1.63)\end{array}$ & $\begin{array}{l}\text { Quartile } 4 \\
(\geq 1.63)\end{array}$ & \\
\hline No. of participants & 4116 & 1027 & 1019 & 1039 & 1031 & \\
\hline Age, mean (SD), years & $58.58(9.03)$ & $57.09(8.75)$ & $58.51(9.05)$ & $59.01(8.93)$ & $59.70(9.17)$ & $<0.001$ \\
\hline Sex, male (\%) & $2187(53.13)$ & $590(57.45)$ & $566(55.54)$ & $530(51.01)$ & $501(48.59)$ & $<0.001$ \\
\hline Education level (\%) & & & & & & 0.89 \\
\hline Illiterate & $1931(46.91)$ & $488(47.52)$ & $486(47.69)$ & $474(45.62)$ & $483(46.85)$ & \\
\hline Primary school & $965(23.45)$ & $239(23.27)$ & $233(22.87)$ & $248(23.87)$ & $245(23.76)$ & \\
\hline Middle school & $853(20.72)$ & $213(20.74)$ & $207(20.31)$ & $212(20.40)$ & $221(21.44)$ & \\
\hline High school or above & $367(8.92)$ & $87(8.47)$ & $93(9.13)$ & $105(10.11)$ & $82(7.95)$ & \\
\hline Residence, urban (\%) & $1237(30.05)$ & $281(27.36)$ & $294(28.85)$ & $316(30.41)$ & $346(33.56)$ & 0.02 \\
\hline Cigarette smoking (\%) & & & & & & $<0.001$ \\
\hline Never smoker & $2510(60.98)$ & $670(65.24)$ & $653(64.08)$ & $614(59.10)$ & $573(55.58)$ & \\
\hline Former smoker & $305(7.41)$ & $55(5.36)$ & $69(6.77)$ & $78(7.51)$ & $103(9.99)$ & \\
\hline Current smoker & $1301(31.61)$ & $302(29.41)$ & $297(29.15)$ & $347(33.40)$ & $355(34.43)$ & \\
\hline Alcohol drinking (\%) & & & & & & 0.09 \\
\hline Never drinker & $2397(58.24)$ & $622(60.56)$ & $595(58.39)$ & $592(56.98)$ & $588(57.03)$ & \\
\hline Former drinker & $308(7.48)$ & $61(5.94)$ & $66(6.48)$ & $91(8.76)$ & $90(8.73)$ & \\
\hline Current drinker & $1411(34.28)$ & $344(33.50)$ & $358(35.13)$ & $356(34.26)$ & $353(34.24)$ & \\
\hline $\mathrm{BMI}$, mean (SD), kg/m² & $22.66(3.39)$ & $21.97(3.00)$ & $22.66(3.30)$ & $22.86(3.37)$ & $23.13(3.76)$ & $<0.001$ \\
\hline WC, mean (SD), cm & $81.35(11.65)$ & $79.40(10.74)$ & $80.95(11.93)$ & $82.22(10.68)$ & $82.80(12.84)$ & $<0.001$ \\
\hline FBG, mean (SD), mg/dl & $102.66(23.79)$ & $100.77(20.67)$ & $103.50(22.93)$ & $102.88(22.87)$ & $103.48(28.00)$ & 0.02 \\
\hline Diastolic BP, mean (SD), mmHg & $73.75(11.69)$ & $72.71(11.41)$ & $73.56(11.91)$ & $74.01(11.59)$ & $74.73(11.76)$ & 0.01 \\
\hline Systolic BP, mean (SD), mmHg & $128.96(51.54)$ & $126.87(48.62)$ & $127.46(43.44)$ & $130.91(64.44)$ & $130.54(46.83)$ & 0.17 \\
\hline TG, mean (SD), mg/dl & $101.93(50.12)$ & $96.76(48.94)$ & $100.86(46.83)$ & $106.56(52.65)$ & $103.45(51.35)$ & $<0.001$ \\
\hline HDL-C, mean (SD), mg/dl & $55.46(14.25)$ & $58.30(15.32)$ & $56.06(14.13)$ & $53.97(13.48)$ & $53.53(13.53)$ & $<0.001$ \\
\hline Hypertension (\%) & $1,266(30.76)$ & $270(26.29)$ & $311(30.52)$ & $324(31.18)$ & $361(35.01)$ & $<0.001$ \\
\hline Dyslipidemia (\%) & $590(14.33)$ & $124(12.07)$ & $120(11.78)$ & $176(16.94)$ & $170(16.49)$ & $<0.001$ \\
\hline Diabetes (\%) & $367(8.92)$ & $78(7.59)$ & $96(9.42)$ & $97(9.34)$ & $96(9.31)$ & 0.40 \\
\hline Heart disease (\%) & $398(9.67)$ & $87(8.47)$ & $90(8.83)$ & $98(9.43)$ & $123(11.93)$ & 0.04 \\
\hline Stroke (\%) & $62(1.51)$ & $17(1.66)$ & $9(0.88)$ & $20(1.92)$ & $16(1.55)$ & 0.26 \\
\hline
\end{tabular}

BMI, body mass index; BP, blood pressure; CHARLS, China Health and Retirement Longitudinal Study; FBG, fasting blood glucose; HDL-C, high-density lipoprotein cholesterol; hs-CRP, high-sensitive C-reactive protein; SD, standard deviation; TG, triglycerides; WC, waist circumference

(2.51, 4.02), 3.34 $(2.56,4.38)$, and $3.34(2.54,4.40)$ times as likely to develop MetS after adjustment for potential confounders (Table 2). Similarly, compared with participants in the lowest group of percent hs-CRP changes, those with percent hs-CRP changes in the second, third and fourth groups were $3.29(2.50,4.37), 3.71(2.78,4.99)$, and $3.76(2.80,5.09)$ times as likely to have MetS. Linear trends were noted in these two sets of analyses $(P$ for trend $<0.001)$. There were no heterogeneities across age, sex and BMI subgroups ( $P$ for interaction $\geq 0.40$; Table 3). Additionally, multiple imputations for missing data did not show appreciable changes in effect estimates across four groups of hs-CRP changes (Additional file 1: Table S3). The effect estimates remained similar after excluding participants with diabetes, heart disease and stroke (Additional file 1: Table S4).

Compared with participants with low baseline/ low increase of hs-CRP, those with high baseline/low increase, low baseline/high increase and high baseline/ high increase were 2.17 (1.52, 3.07), 2.55 (2.04, 3.21) and $2.68(1.40,4.90)$ times as likely to have MetS ( $P$ for trend $<0.001$, Additional file 1: Fig. S3).

\section{Association of baseline hs-CRP and hs-CRP changes with MetS components}

Participants in the highest quartile of hs-CRP had a higher likelihood of elevated WC (multivariableadjusted OR, 1.46; 95\% CI, 1.10, 1.93), elevated TG (1.32; 
Table 2 Association of baseline hs-CRP and longitudinal hs-CRP changes with incident MetS $(\mathrm{N}=4,116)$

\begin{tabular}{|c|c|c|c|c|}
\hline Models & Cases / total (\%) & $\begin{array}{l}\text { Model } 1 \\
\text { OR }(95 \% \mathrm{Cl})\end{array}$ & $\begin{array}{l}\text { Model } 2 \\
\text { OR }(95 \% \mathrm{Cl})\end{array}$ & $\begin{array}{l}\text { Model } 3 \\
\text { OR }(95 \% \mathrm{Cl})\end{array}$ \\
\hline \multicolumn{5}{|l|}{ Hs-CRP quartiles at baseline } \\
\hline Quartile $1(<0.48)$ & $80 / 1,027(13.85)$ & 1.00 (Ref.) & 1.00 (Ref.) & 1.00 (Ref.) \\
\hline Quartile $2(\geq 0.48$ to $<0.81)$ & $130 / 1,019(12.43)$ & $1.73(1.29,2.33)$ & $1.73(1.29,2.33)$ & $1.51(1.12,2.06)$ \\
\hline Quartile 3 ( $\geq 0.81$ to $<1.63$ ) & $144 / 1,039(12.35)$ & $1.90(1.43,2.55)$ & $1.91(1.44,2.56)$ & $1.50(1.11,2.04)$ \\
\hline Quartile 4 ( $\geq 1.63)$ & 181/1,031 (13.32) & $2.52(1.91,3.35)$ & $2.53(1.92,3.37)$ & $1.83(1.37,2.47)$ \\
\hline$P$ for trend $d^{a}$ & $535 / 4,116(13.00)$ & $<0.001$ & $<0.001$ & $<0.001$ \\
\hline \multicolumn{5}{|l|}{ Log (hs-CRP) level } \\
\hline $\begin{array}{l}\text { Each } 1 \mathrm{mg} / \mathrm{L} \text { increase } \\
\text { levated hs-CRP b }\end{array}$ & $535 / 4,116(13.00)$ & $1.39(1.26,1.55)$ & $1.40(1.27,1.56)$ & $1.23(1.10,1.38)$ \\
\hline No & $370 / 3,668(10.09)$ & 1.00 (Ref.) & 1.00 (Ref.) & 1.00 (Ref.) \\
\hline Yes & 78/448 (17.41) & $1.48(1.13,1.92)$ & $1.50(1.14,1.94)$ & $1.27(0.95,1.67)$ \\
\hline \multicolumn{5}{|c|}{$\begin{array}{l}\text { Groups of absolute changes in hs-CRP } \\
\text { during the follow-upc }\end{array}$} \\
\hline Group1 $(\leq 0)$ & $140 / 1,479(9.47)$ & 1.00 (Ref.) & 1.00 (Ref.) & 1.00 (Ref.) \\
\hline Group $2(>0$ to $\leq 0.41)$ & 77/873 (8.82) & $1.33(0.96,1.82)$ & $2.44(1.84,3.24)$ & $3.24(2.51,4.02)$ \\
\hline Group $3(>0.41$ to $\leq 1.21)$ & 133/868 (15.32) & $1.30(0.93,1.80)$ & $2.41(1.80,3.24)$ & $3.34(2.56,4.38)$ \\
\hline Group 4 (> 1.21) & 185/896 (20.65) & $1.31(0.94,1.82)$ & $2.51(1.87,3.39)$ & $3.34(2.54,4.40)$ \\
\hline$P$ for trend ${ }^{\mathrm{a}}$ & $535 / 4,116(13.00)$ & $<0.001$ & $<0.001$ & $<0.001$ \\
\hline \multicolumn{5}{|c|}{$\begin{array}{l}\text { Groups of percent changes in hs-CRP dur- } \\
\text { ing the follow-up }\end{array}$} \\
\hline Group $1(\leq 0)$ & $140 / 1,479(9.47)$ & 1.00 (Ref.) & 1.00 (Ref.) & 1.00 (Ref.) \\
\hline Group 2 (>0 to $\leq 60 \%)$ & 113/870 (12.99) & $1.98(1.49,2.64)$ & $2.26(1.69,3.01)$ & $3.29(2.50,4.37)$ \\
\hline Group 3 (>60\% to $\leq 181 \%$ ) & 120/871 (13.78) & $1.87(1.39,2.52)$ & $2.22(1.65,3.00)$ & $3.71(2.78,4.99)$ \\
\hline Group 4 (> 181\%) & $162 / 996(16.27)$ & $1.91(1.41,2.58)$ & $2.24(1.65,3.03)$ & $3.76(2.80,5.09)$ \\
\hline$P$ for trend ${ }^{\mathrm{a}}$ & $535 / 4,116(13.00)$ & $<0.001$ & $<0.001$ & $<0.001$ \\
\hline
\end{tabular}

Model 1: Non-adjusted

Model 2: Adjusted for age (continuous, years), sex (male and female), education level (illiterate, primary school, middle school, and high school or above), and residence (urban and rural)

Model 3: Adjusted for the variables in Model 2 and cigarette smoking (never, former, and current), alcohol drinking (never, former, and current), BMI (continuous, kg/ $\mathrm{m}^{2}$ ), hypertension (yes and no), dyslipidemia (yes and no), diabetes (yes and no), heart disease (yes and no) and stroke (yes and no)

Baseline hs-CRP (continuous, mg/L) was additionally adjusted for as a covariate in the analysis of quartiles of absolute changes (or percent changes) in SUA during the follow-up

$\mathrm{Cl}$, confidence interval; hs-CRP, high-sensitive C-reactive protein; MetS, metabolic syndrome; OR, odds ratio

a $P$ values for trend were estimated by modelling serum hs-CRP using the median for each category

${ }^{b}$ Elevated hs-CRP was categorized into two groups: yes (higher than $3 \mathrm{mg} / \mathrm{L}$ ) and no (lower than $3 \mathrm{mg} / \mathrm{L}$ )

c Participants were categorized into four groups: Group 1 included participants with negative or null changes, and Group 2-4 were tertiles for the remaining participants

d Participants were categorized into four groups: Group 1 included participants with negative or null percent changes, and Group 2-4 were tertiles for the remaining participants

$1.06,1.66)$ and elevated FBG $(1.58 ; 1.18,2.12$; Table 4$)$, compared with those in the lowest quartile of hs-CRP. Participants in the group of highest hs-CRP changes had a higher likelihood of elevated WC (multivariable-adjusted OR, 1.72; 95\% CI 1.32, 2.24), elevated TG (3.08; 2.50, 3.80), elevated HDL-C (3.50; $2.57,4.79)$, and elevated FBG (1.60; 1.25, 2.04; Table 4), compared with participants in the group of lowest hs-CRP changes. Additionally, there were no heterogeneities across age, sex and BMI subgroups for the association between
hs-CRP and MetS components ( $P$ for interaction $\geq 0.19$; Additional file 1: Table S5). There were generally no heterogeneities across age, sex, and BMI subgroups for the association between hs-CRP changes and MetS components ( $P$ for interaction $\geq 0.09)$, except for sex $(P$ for interaction $=0.04)$ and BMI $(P$ for interaction $<0.001)$ subgroups for elevated WC, age subgroups ( $P$ for interaction $=0.01)$ for elevated TG, and age subgroups ( $P$ for interaction $=0.02$; Additional file 1: Table S6) for elevated BP. 


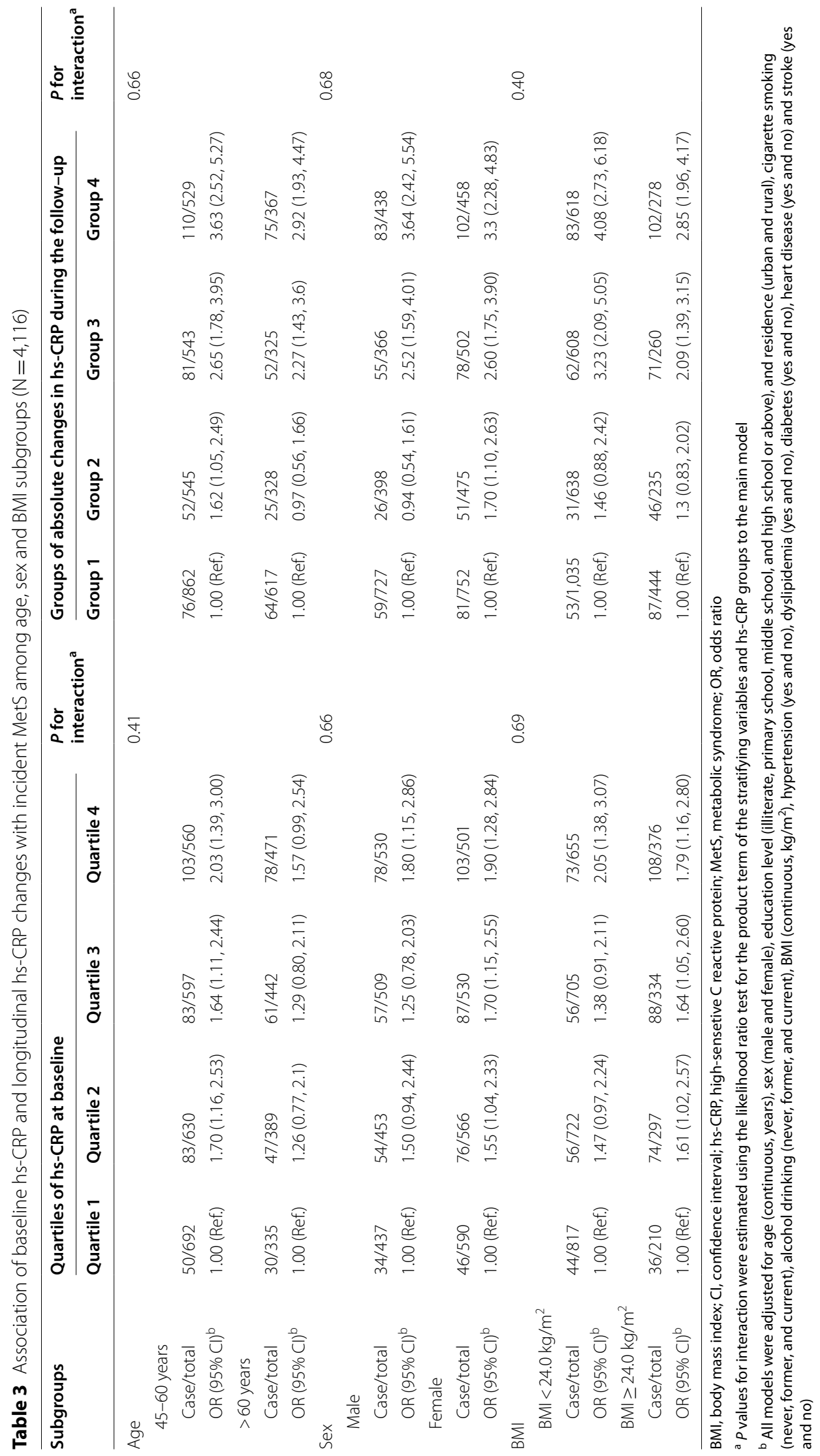




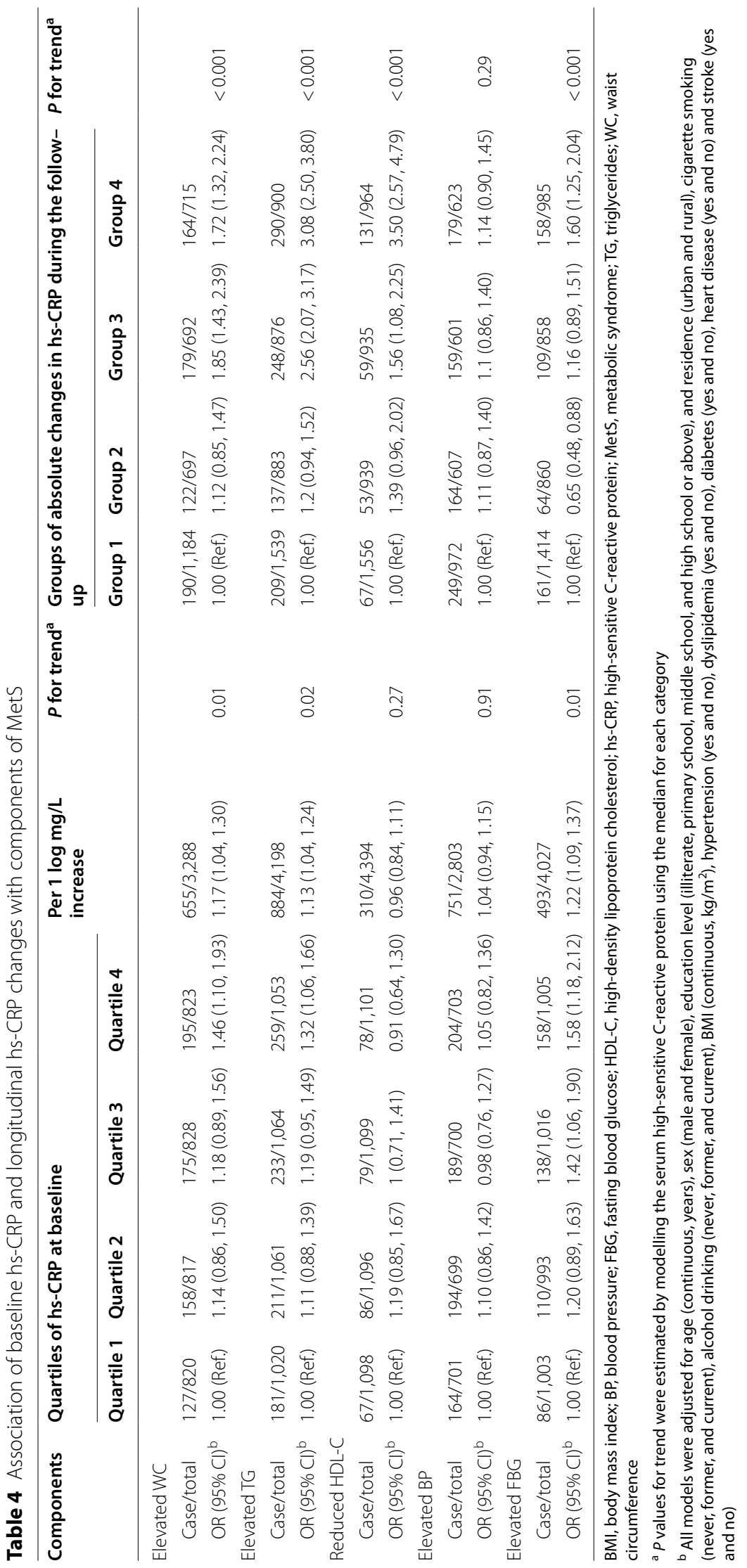




\section{Author (Year and Country)}

A) Highest and lowest hs-CRP
Xue (2021, China)
Hong (2020, China)
Yoon (2018, Korea)
Lim (2005, Korea)
Laaksonen (2004, Finland)
Han (2002, Mexico)
Random effects model

RR $\quad 95 \% \mathrm{Cl}$

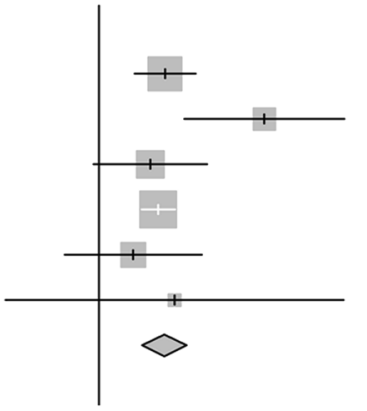

$1.64[1.31 ; 2.06]$

$3.45[1.90 ; 6.27]$

$1.47[0.96 ; 2.25]$

$1.56[1.38 ; 1.76]$

$1.29[0.77 ; 2.16]$

$1.76[0.50 ; 6.22]$

$1.63[1.38 ; 1.93]$

B) Per $1 \log ($ hs-CRP) unit increment

Xue (2021, China)

Saisho (2013, Japan)

Oda (2013, Japan)

Musani (2013, US)

Bo (2009, Italy)

Random effects model

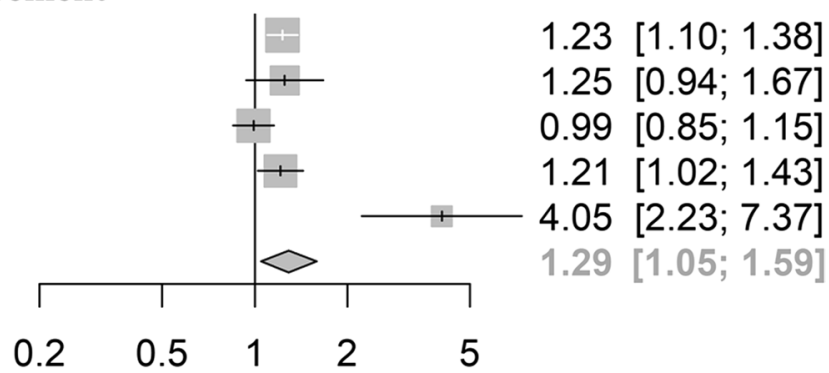

Fig. 1 Meta-analysis for the association between hs-CRP and incident metabolic syndrome. A) Pooled relative risk of incident metabolic syndrome compared the highest and lowest serum hs-CRP; and B) Pooled relative risk of incident metabolic syndrome with log-transformed serum hs-CRP. Cl, confidence interval; RR, relative rask; hs-CRP, high-sensitive C-reactive protein

\section{Meta-analysis for the association between CRP and MetS}

We identified 9,902 records, including 3,980 from PubMed and 5,922 from Embase. We excluded 2,514 duplicate records, and 7,324 records based on title and abstract. Of the 64 records with full texts, we excluded 49 with non-cohort studies, 5 with non-original research ( 1 review, 2 conference abstracts, and 2 letters to editor), and 1 record without reporting the effect estimates. Thus a total of 10 studies (including the current one) were eligible for our meta-analysis (Additional file 1: Fig. S2).

The 10 studies comprised a total of 24,552 participants and 4,209 incident MetS cases [4-7, 11, 12, 23-25], of which five studies treated the CRP level as categorical variable, and four studies treated the CRP level as continuous variable with log transformation, and our current study in Chinese treated the CRP as both categorical and continuous variables (Additional file 1: Table 7). The Newcastle-Ottawa score for included studies ranged from 5 to 8 (out of 8 ), with 5 studies having moderate quality (6-7 points) and 3 studies having high quality ( 8 points) (Additional file 1: Table S8).

The pooled RR (95\% CI) for incident MetS comparing individuals in the highest versus lowest groups was 1.63 (95\% CI 1.38, 1.93; $I^{2}=32.4 \%, P=0.01$; Fig. 1 ). The pooled RR (95\% CI) for incident MetS for each 1 SD increase in $\log$ CRP was 1.29 (95\% CI 1.05, 1.59; $I^{2}=82.0 \%, P<0.001$;
Fig. 1). The sensitivity analyses with exclusions of a study each time further confirmed the robustness of the results in our meta-analyses (Additional file 1: Fig. S4). There was no evidence of publication bias, as $P$ values for Egger's test were 0.51 and 0.22 for two meta-analyses (Additional file 1: Fig. S5).

\section{Discussion}

In our nationwide population-based study, we found associations of higher baseline hs-CRP levels and hsCRP increases with elevated risks of MetS among middle-aged and elderly Chinese population during a 4-year follow-up. Of participant groups defined by baseline and changes of hs-CRP, the group with high baseline/high increase had the highest risk of incident MetS. No heterogeneities were found in subgroups stratified by age, sex and BMI. In addition, our results were further confirmed by the meta-analysis of 10 cohort studies.

In our study, participants in the highest quartile of hs-CRP had $83 \%$ increased odds of having MetS compared with those in the lowest quartile, and there was a linear trend across quartiles. Our findings were generally consistent with those from another recent study that reported a positive association between hs-CRP and MetS among 886 Chinese adults (average age, 52.0 years) during a 5-year follow-up [6]. However, this study had a 
relatively small sample size and was limited to residents in a small town in China. Using a nationwide populationbased design, we were able to improve the robustness and generalizability of these findings in China. Similarly, one study in 9773 Korean adults aged 40-65 years [7] showed that high hs-CRP was an independent predictor for incident MetS in Asia. Consistently, two smaller studies from US (1,215 participants) [4] and Italy (201 participants) [5] also reported positive associations between hs-CRP and incident MetS. While the magnitude of association was equivalent between men and women in our study (OR, 1.80 vs 1.90 ), sex heterogeneity was reported before. Two studies in Chinese adults [6] and Mexicans [23] reported a positive association in women but not men in subgroup analyses. Although higher visceral fat levels [26] and sex hormone estrogen [27] in women could potentially contribute to MetS risk, the sex heterogeneity should be further examined in more large population-based cohort studies worldwide.

In addition, one novel finding of our work was the strong positive association between longitudinal increases in hs-CRP and incident MetS. Previous studies using only a single measurement of hs-CRP to represent chronic inflammation and its relationship with MetS ignored the time-varying nature of hs-CRP. Our study with two repeated measures of hs-CRP during a fouryear follow-up further confirmed the positive association between hs-CRP increase and MetS risk. To our knowledge, our study has been the first to explore the relationship between hs-CRP dynamics and incident MetS in Chinese adults. Consistent with our work, a community-based cohort study among 10,160 participants in the US showed that participants with large increases or sustained elevations in hs-CRP over a 6-year period had 1.39 to 1.56 times higher risks of incident diabetes [8]. In addition, our analyses in examining the combined effects of baseline and changes of hs-CRP showed that the participants with high baseline/high increase had the highest risk of MetS among participant categories. In agreement with our finding, a separate study among 6,439 Chinese adults used latent mixture modeling to identify differential hs-CRP trajectories based on three measurements in a three-year follow-up, and demonstrated that participants in the "moderate-increased" hs-CRP group had 2.18-fold higher risk of incident diabetes, compared with those in the "low-stable" hs-CRP group [9]. Ideally, multiple repeated measurements should be collected in large cohort studies to confirm the association between hsCRP dynamics and MetS in future.

Our meta-analysis of 10 cohort studies among 24,552 participants supported a positive association between hs-CRP and incident MetS. In particular, summary effect estimates based on both categorical and continuous variables of hs-CRP consistently confirmed the positive association. However, overall heterogeneity was observed in our meta-analysis, which could be attributable to differences in participant characteristics (such as age and sex) and research designs (such as follow-up durations and sample sizes). However, due to the small number of original studies, we did not statistically explore the subgroup heterogeneity by meta-regression. The findings from our cohort study and meta-analysis suggest that individuals with increased hs-CRP levels might need to be closely monitored for future risk of MetS in clinical practice. Of note, previous studies proposed to incorporate hs-CRP into the criteria for defining MetS, given the added value of hs-CRP for predicting incident cardiovascular diseases [28] and mortality [29]. Thus future large-scale population-based studies are still warranted to examine whether hs-CRP should be included as one component of MetS.

While the underlying mechanisms for the association between increased hs-CRP and MetS are evolving, there are certain possible explanations. First, CRP is an acute-phase reactant by the liver in response to proinflammation cytokines such as interleukin- 6 and tumor necrosis factor- $\alpha$ [30]. Besides stimulating the synthesis of CRP in liver, these cytokines cause abnormalities in lipid metabolism [31] and glucometabolism [32], leading to the development of MetS. Second, CRP has an impact on insulin-stimulated phosphorylation of insulin receptor substrate 1 in vitro, suggesting that human CRP could disturb the glucose metabolism via insulin signaling pathways that regulate cell glucose transport [33]. Third, increased CRP might reduce nitric oxide bioavailability via decreasing urinary excretion of cyclic guanosine monophosphate and activating NADPH oxidase, which could aggravate hypertension and thus MetS [34, 35].

Our study has major strengths including a large sample size, a prospective population-based design, repeated measurements of hs-CRP, and a comprehensive metaanalysis. Our study has also been the first to explore the association between longitudinal changes in hs-CRP and incident MetS in China. However, some limitations should be acknowledged in our original analyses. First, a large number of participants were excluded from final analyses due to data missing and loss to the follow-up, which could lead to selection and information bias. Second, reverse causality could not be eliminated due to a short follow-up. However, our finding was corroborated by studies with longer follow-ups in other populations. Third, we failed to adjust for certain potential confounders such as physical activity, detailed medical conditions and medications due to data unavailability, so residual confounding could not be avoided. Fourth, as our study was conducted in middle-aged and elderly Chinese, our 
findings may not be readily generalizable to the entire Chinese population or other populations, especially the younger adults. However, our meta-analysis of multiple studies in a wider age range lends support to the generalizability of our findings.

\section{Conclusion}

In conclusion, we found positive associations of baseline hs-CRP and longitudinal hs-CRP changes with incident MetS, and such positive associations were confirmed by additional meta-analyses. Our findings suggest that individuals with high hs-CRP levels may need to be closely monitored for future risk of MetS, and highlight the need to examine whether high hs-CRP levels should be incorporated into the components for MetS.

\section{Abbreviations}

BMI: Body mass index; BP: Blood pressure; CHARLS: China Health and Retirement Longitudinal Study; Cl: Confidence interval; FBG: Fasting blood glucose; HDL-C: High-density lipoprotein cholesterol; HR: Hazard ratio; Hs-CRP: Highsensitive C-reactive protein; MetS: Metabolic syndrome; OR: Odds ratio; RR: Relative risk; SD: Standard deviation; TG: Triglycerides; WC: Waist circumference.

\section{Supplementary Information}

The online version contains supplementary material available at https://doi. org/10.1186/s12986-021-00632-6.

Additional file 1. Table S1: Search strategy for PubMed and EMBASE for the meta-analysis; Table S2: Comparison of 4116 study participants included in final analyses and 2687 excluded due to data missing in the CHARLS; Table S3: Association of baseline hs-CRP and longitudinal hs-CRP changes with incident MetS after multiple imputations of missing data ( $N$ $=4497) ;$ Table S4: Association of baseline hs-CRP and longitudinal hs-CRP changes and incident MetS after excluding participants with diabetes, heart disease and stroke $(\mathrm{N}=3561)$; Table S5: Association between baseline hs-CRP and components of MetS among age, sex and BMI subgroups ( $N=4116)$; Table S6: Association between longitudinal hs-CRP changes and components of MetS among age, sex and BMI subgroups; Table S7: Basic information of studies included in the meta-analysis; Table S8: Quality assessment for the 10 included studies in the meta-analysis; Figure S1: Flowchart of inclusion and exclusion of study participants; Figure S2: Flowchart of literature identification for the meta-analysis; Figure S3: Association of combined baseline hs-CRP and longitudinal hs-CRP changes with incident MetS; Figure S4: Sensitivity analyses for the association between hs-CRP and incident MetS with exclusions of each study a time; Figure S5: Funnel plot for assessment of publication bias for the association between hs-CRP and MetS.

\section{Acknowledgements}

We thank the China Center for Economic Research, National School of Development at Peking University for providing the CHARLS data.

\section{Authors' contributions}

XFP, QX and TY designed the study. QX and XY analyzed the data. QX drafted the first manuscript. XY, YH, DZ, YW, YW, JZ, YL, CXY, JP, TY, and XFP provided critical revision of the manuscript for important intellectual content. All authors contributed to the interpretation of the data and approved the final version of the manuscript. All authors read and approved the final manuscript.

\section{Funding}

The study was supported by the Chengdu High-level Key Clinical Specialty Construction Project. The funding agencies had no role in design, analysis, interpretation, or writing of this study.

\section{Availability of data and materials}

The datasets used in the current study are available on the CHARLS official website, http://charls.pku.edu.cn/. All data, analytic methods, and study materials presented within this article are available from the corresponding authors on reasonable request.

\section{Declarations}

\section{Ethics approval and consent to participate}

The study was approved by the Biomedical Ethics Review Committee of Peking University (IRB00001052-11015), and informed consent was obtained from all participants.

\section{Consent for publication}

Not applicable.

\section{Competing interests}

The authors declare that they have no competing interests.

\section{Author details}

${ }^{1}$ Department of Epidemiology and Biostatistics, School of Public Health, Chengdu Medical College, Chengdu, Sichuan, China. ${ }^{2}$ HEOA Group, Institute for Healthy Cities and West China Research Center for Rural Health Development, Sichuan University, Chengdu, Sichuan, China. ${ }^{3}$ Department of Epidemiology and Biostatistics, West China School of Public Health and West China Fourth Hospital, Sichuan University, Chengdu, Sichuan, China. ${ }^{4}$ Department of Cardiology, Shunde Hospital, Southern Medical University, Foshan, Guangdong, China. ${ }^{5}$ The George Institute for Global Health, Faculty of Medicine, University of New South Wales, Sydney, NSW, Australia. ${ }^{6}$ Center for Health Management and Policy Research, School of Public Health, Cheeloo College of Medicine, Shandong University, Jinan, Shandong 250012, China. ${ }^{7}$ Department of Epidemiology and Biostatistics, Ministry of Education Key Laboratory of Environment and Health and State Key Laboratory of Environmental Health (Incubation), School of Public Health, Tongji Medical College, Huazhong University of Science and Technology, Wuhan, Hubei, China. ${ }^{8}$ Department of Communicable Diseases Control and Prevention, Shenzhen Center for Disease Control and Prevention, Shenzhen, Guangdong, China. ${ }^{9}$ The Ministry of Education - Shanghai Key Laboratory of Children's Environmental Health, Xinhua Hospital, Shanghai Jiao Tong University School of Medicine, Shanghai, China. ${ }^{10} \mathrm{Center}$ of Gastrointestinal and Minimally Invasive Surgery, Department of General Surgery, The Third People's Hospital of Chengdu and The Affiliated Hospital of Southwest Jiaotong University, Chengdu, Sichuan, China. ${ }^{11}$ Center for Obesity and Metabolic Health, The Third People's Hospital of Chengdu and The Affiliated Hospital of Southwest Jiaotong University, Chengdu, Sichuan, China. ${ }^{12}$ Key Laboratory of Birth Defects and Related Diseases of Women and Children (Sichuan University), Ministry of Education, West China Second University Hospital, Sichuan University, Chengdu, Sichuan, China.

Received: 15 September 2021 Accepted: 30 November 2021 Published online: 06 January 2022

\section{References}

1. Alberti KGM, Zimmet P, Shaw J. The metabolic syndrome-a new worldwide definition. Lancet. 2005;366:1059-62.

2. Cornier M-A, Dabelea D, Hernandez TL, Lindstrom RC, Steig AJ, Stob NR, Van Pelt RE, Wang H, Eckel RH. The metabolic syndrome. Endocr Rev. 2008;29:777-822.

3. Pepys MB, Hirschfield GM. C-reactive protein: a critical update. J Clin Investig. 2003;111:1805-12.

4. Musani SK, Vasan RS, Bidulescu A, Liu J, Xanthakis V, Sims M, Gawalapu RK, Samdarshi TE, Steffes M, Taylor HA, Fox ER. Aldosterone, c-reactive protein, and plasma b-type natriuretic peptide are associated with 
the development of metabolic syndrome and longitudinal changes in metabolic syndrome components: findings from the jackson heart study. Diabetes Care. 2013;36:3084-92.

5. Bo S, Rosato R, Ciccone G, Gambino R, Durazzo M, Gentile L, Cassader M, Cavallo-Perin P, Pagano G. What predicts the occurrence of the metabolic syndrome in a population-based cohort of adult healthy subjects? Diabetes Metab Res Rev. 2009;25:76-82.

6. Hong GB, Gao PC, Chen YY, Xia Y, Ke XS, Shao XF, Xiong CX, Chen HS, Xiao $\mathrm{H}$, Ning J, Zou HQ. High-sensitivity c-reactive protein leads to increased incident metabolic syndrome in women but not in men: a five-year follow-up study in a Chinese population. Diabetes Metab Syndr Obes Targets Ther. 2020;13:581-90.

7. Lim S, Lee HK, Kimm KC, Park C, Shin C, Cho NH. C-reactive protein level as an independent risk factor of metabolic syndrome in the Korean population CRP as risk factor of metabolic syndrome. Diabetes Res Clin Pract. 2005;70:126-33.

8. Parrinello CM, Lutsey PL, Ballantyne CM, Folsom AR, Pankow JS, Selvin E. Six-year change in high-sensitivity C-reactive protein and risk of diabetes, cardiovascular disease, and mortality. Am Heart. 2015;170:380-389.e384.

9. Xu R, Jiang X, Fan Z, Wan Y, Gao X. The trajectory of high sensitivity C-reactive protein is associated with incident diabetes in Chinese adults. Nutr Metab. 2020;17:1-8.

10. Li R, Li W, Lun Z, Zhang H, Sun Z, Kanu JS, Qiu S, Cheng Y, Liu Y. Prevalence of metabolic syndrome in Mainland China: a meta-analysis of published studies. BMC Public Health. 2016;16:1-10.

11. Yoon K, Ryu S, Lee J, Park JD. Higher and increased concentration of hs-CRP within normal range can predict the incidence of metabolic syndrome in healthy men. Diabetes Metab Syndr. 2018;12:977-83.

12. Oda E. High-sensitivity C-reactive protein and white blood cell count equally predict development of the metabolic syndrome in a Japanese health screening population. Acta Diabetol. 2013;50:633-8.

13. Zhao Y, Hu Y, Smith JP, Strauss J, Yang G. Cohort profile: the China health and retirement longitudinal study (CHARLS). Int J Epidemiol. 2014;43:61-8

14. Mahajan A, Jaiswal A, Tabassum R, Podder A, Ghosh S, Madhu S, Mathur SK, Tandon N, Bharadwaj D. Elevated levels of C-reactive protein as a risk factor for metabolic syndrome in Indians. Atherosclerosis. 2012;220:275-81.

15. Zhu JR, Gao RL, Zhao SP, Guoping LU, Zhao D, Jianjun LI, Zhu JR, Gao RL, Zhao SP, Guoping LU. 2016 Chinese guidelines for the management of dyslipidemia in adults. J Geriatr Cardiol JGC. 2018;15:1-29.

16. Shen $Y$, Zhang $Y$, Xiong $S$, Zhu X, Ke C. High-sensitivity C-reactive protein and cystatin C independently and jointly predict all-cause mortality among the middle-aged and elderly Chinese population. Clin Biochem. 2019;65:7-14

17. Zhao Y, Crimmins EM, Hu P, Shen Y, Smith JP, Strauss J, Wang Y, Zhang Y. Prevalence, diagnosis, and management of diabetes mellitus among older Chinese: results from the China Health and Retirement Longitudinal Study. Int J Public Health. 2016;61:347-56.

18. Ning M, Zhang Q, Yang M. Comparison of self-reported and biomedical data on hypertension and diabetes: findings from the China Health and Retirement Longitudinal Study (CHARLS). BMJ Open. 2016;6:e009836.

19. Allison MA, Budoff MJ, Wong ND, Blumenthal RS, Schreiner PJ, Criqui MH. Prevalence of and risk factors for subclinical cardiovascular disease in selected US Hispanic ethnic groups: the Multi-Ethnic Study of Atherosclerosis. Am J Epidemiol. 2008;167:962-9.

20. Pan X-F, Wang L, Pan A. Epidemiology and determinants of obesity in China. Lancet Diabetes Endocrinol. 2021:9:373-92.

21. Wells G, Shea B, O'Connell D, Peterson J, Welch V, Losos M, Tugwell P. Newcastle-Ottawa quality assessment scale cohort studies. 2014.

22. Zhang J, Kai FY. What's the relative risk? A method of correcting the odds ratio in cohort studies of common outcomes. JAMA. 1998:280:1690-1.

23. Han TS, Sattar N, Williams K, Gonzalez-Villalpando C, Lean MEJ, Haffner SM. Prospective study of $\mathrm{C}$-reactive protein in relation to the development of diabetes and metabolic syndrome in the Mexico City diabetes study. Diabetes Care. 2002;25:2016-21.

24. Laaksonen DE, Niskanen L, Nyyssönen K, Punnonen K, Tuomainen TP, Valkonen VP, Salonen R, Salonen JT. C-reactive protein and the development of the metabolic syndrome and diabetes in middle-aged men. Diabetologia. 2004;47:1403-10.
25. Saisho Y, Hirose H, Roberts R, Abe T, Kawabe H, Itoh H. C-reactive protein, high-molecular-weight adiponectin and development of metabolic syndrome in the Japanese general population: a longitudinal cohort study. PLOS ONE. 2013;8:e73430.

26. Blouin K, Boivin A, Tchernof A. Androgens and body fat distribution. J Steroid Biochem Mol Biol. 2008;108:272-80.

27. Straub RH. The complex role of estrogens in inflammation. Endocr Rev. 2007;28:521-74.

28. Suzuki T, Katz R, Jenny NS, Zakai NA, LeWinter MM, Barzilay Jl, Cushman M. Metabolic syndrome, inflammation, and incident heart failure in the elderly: the cardiovascular health study. Circ Heart Fail. 2008;1:242-8.

29. Suzuki T, Voeks J, Zakai NA, Jenny NS, Brown TM, Safford MM, LeWinter M, Howard G, Cushman M. Metabolic syndrome, C-reactive protein, and mortality in US Blacks and Whites: the Reasons for Geographic and Racial Differences in Stroke (REGARDS) study. Diabetes Care. 2014;37:2284-90.

30. Ansar W, Ghosh S. C-reactive protein and the biology of disease. Immunol Res. 2013;56:131-42.

31. Maury E, Brichard S. Adipokine dysregulation, adipose tissue inflammation and metabolic syndrome. Mol Cell Endocrinol. 2010;314:1-16.

32. McCracken E, Monaghan M, Sreenivasan S. Pathophysiology of the metabolic syndrome. Clin Dermatol. 2018;36:14-20.

33. D'Alessandris C, Lauro R, Presta I, Sesti G. C-reactive protein induces phosphorylation of insulin receptor substrate-1 on Ser 307 and Ser 612 in L6 myocytes, thereby impairing the insulin signalling pathway that promotes glucose transport. Diabetologia. 2007;50:840-9.

34. Pravenec M, Kajiya T, Zídek V, Landa V, Mlejnek P, Šimáková M, Šilhavý J, Malínská H, Oliyarnyk O, Kazdová L. Effects of human C-reactive protein on pathogenesis of features of the metabolic syndrome. Hypertension. 2011:57:731-7.

35. Jialal I, Verma S, Devaraj S. Inhibition of endothelial nitric oxide synthase by C-reactive protein: clinical relevance. Oxford: Oxford University Press; 2009.

\section{Publisher's Note}

Springer Nature remains neutral with regard to jurisdictional claims in published maps and institutional affiliations.

Ready to submit your research? Choose BMC and benefit from

- fast, convenient online submission

- thorough peer review by experienced researchers in your field

- rapid publication on acceptance

- support for research data, including large and complex data types

- gold Open Access which fosters wider collaboration and increased citations

- maximum visibility for your research: over $100 \mathrm{M}$ website views per year

At BMC, research is always in progress.

Learn more biomedcentral.com/submissions 